\title{
The influence of two varieties of proprioceptive information on judgments of the spatial position of an object while the visual input is transformed
} G. SINGER, SCHOOL OF BEHAVIOURAL SCIENCES,
MACQUARIE UNIVERSITY, North Ryde, N. S.W., Australia

This experiment was concerned with the effects of two varieties of proprioceptive activity on an experimentally induced intersensory conflict between vision and proprioception. Ss who were required to make contact with a bar while the visual input from the bar was transformed showed no difference in their subsequent judgments of horizontality of the bar compared to Ss who had no such prior contact. Over 10 trials, Ss showed a significant increase in the degree to which later responses corresponded with the transformed visual input.

If before entering the eye light is spatially transformed by means of an optical device (i.e., mirrors, prisms, etc.) visually guided behavior such as reaching for, or pointing at, objects and making judgments of the spatial position and orientation of objects is disrupted. The essential feature of behavior following this disruption is a change in the direction of the responses such that compensation is made for the effects of the transformation. The compensation is not necessarily in the direction of accuracy or of inaccuracy but depends on the nature of the task. This change in behavior has been referred to as sensory motor adaptation (Held \& Freedman, 1963), or behavioral compensation (Day \& Singer, 1967).

In these situations of intersensory discordance or conflict, compensation is usually in the direction of the visual input. Harris (1965) has argued that visual dominance is such that the felt position of a limb changes in the direction of visual transformation. Over (1966) has shown that the extent of visual dominance over the proprioceptive system of the arm is a function of the degree of discordance between the two systems, e.g., a Dove prism system, transforming the visual input so that the retinal projection of a physically horizontal bar is slanted $15 \mathrm{deg}$ from the horizontal, will lead to a proprioceptive response which is almost completely in accordance with this visual transformation. As the transformation of the visual input is changed so that the discordance between vision and proprioception is increased, the extent of visual dominance is reduced and with a 90 deg discordance the proprioceptive response seems unaffected by the visual transformation. Over (1966) has also shown that the extent of visual dominance does not change during 10 consecutive trials, i.e., Ss' responses which were initially inaccurate in objective terms (in the direction of visual transformation) remained so.

The purpose of the present experiment was to determine whether the introduction of a greater variety of proprioceptive activity than that used by Over (1966), relating active movement and degree of spatial conjunction, will lead to a resolution of the intersensory conflict in the direction of proprioception as has been suggested by Over (1966).

Apparatus. The apparatus was a modification of a system used earlier (Singer \& Day, 1966; Collins \& Singer, in press) in which the relative angular orientations of two Dove prisms achieved a transformation of the visual input so that the retinal projection of a physically horizontal bar was slanted $20 \mathrm{deg}$ or $40 \mathrm{deg}$ up or down on Ss left. The orientation of the bar could be judged both visually, by viewing monocularly through an eyepiece with the chin on a chin rest, and proprioceptively, by motion of the hand across its upper edge. $A$ rotary control was used by $S$ to vary the orientation of the bar about its central pivot so that it appeared horizontal. A protractor scale allowed measurements of bar-orientation to the nearest $0.25 \mathrm{deg}$. During the pretest, when judgments of horizontality were made proprioceptively, vision was occluded by blocking the eyepiece.

Subjects. There were four groups of 10 male and female Ss each, recruited from a third year course in psychology. Ss were alternately assigned to groups on reporting.
Procedure. Each trial consisted of four pretests and a $5 \mathrm{~min}$ exposure period. During the pretest $S$ adjusted the bar to apparent horizontal proprioceptively without vision from four starting positions, $\pm 2.5 \mathrm{deg}$ and \pm 5 deg, where the plus and minus signs indicate upward and downward, respectively on S's left. The order of presentation of these starting positions was varied for each $S$ by a random allocation procedure.

There were four exposure conditions; for two conditions the prisms were adjusted so that the retinal projection of the physically horizontal bar was slanted $20 \mathrm{deg}$, and for the other two conditions slanted $40 \mathrm{deg}$, while it was viewed through the eyepiece. For half the $S s$ in each condition the bar was seen as slanted down on S's left, and for other half up on S's left. In all four conditions $S$ was required to adjust the bar 10 times at $1 / 2 \mathrm{~min}$ intervals so that it felt horizontal, while moving his right hand across the bar and looking at it through the eyepiece, operating the rotary control with his left hand. For two of the slant conditions (A) (20 deg and $40 \mathrm{deg}$ ), $S$ was required to place his hand on the bar before each adjustment while viewing it through the eyepiece; and for the other two slant conditions (B) (20 deg and $40 \mathrm{deg}$ ) E placed S's hand on the bar before each adjustment, and $S$ was then immediately allowed to look through the eyepiece. Thus, in Conditions A 20 deg and $40 \mathrm{deg} S$ had to make contact with the bar while being exposed to the transformed visual input, before making each of the 10 adjustments, whereas in Conditions B 20 deg and 40 deg S's hand was already on the bar when the transformed visual field was exposed for making each of the 10 adjustments. Between adjustments $S$ rested his hand on the table while the eyepiece was blocked.

Results. The difference between the mean of the four pretests and each of the 10 adjustments was S's adaptation measure. Only two of the 400 measures were in an unexpected direction (in the direction not opposite to the prism transformation).

The means of these adaptation measures for each of the four conditions on all 10 trials are shown in Table 1 . The contrast between Conditions $A$ and Conditions $B$ is not significant, $F=2.5$, $\mathrm{df}=1 / 39, \mathrm{p}<.05$ (Rodger, 1967). A trend analysis of the means of Table 1 shows a significant linear trend, for three of the four experimental conditions. (For Conditions A 20, $\mathrm{F}=7.16$, $\mathrm{df}=$ $1 / 9 ; \mathrm{A} 40, \mathrm{~F}=6.01, \mathrm{df}=1 / 9 ;$ and $\mathrm{B} 40, \mathrm{~F}=7.75, \mathrm{df}=1 / 9, \mathrm{p}<$ .05 ; but not for $\mathrm{B} 20, \mathrm{~F}=0.7, \mathrm{df}=1 / 9, \mathrm{p}>.05$.) Thus under both conditions of exposure there is an increasing proprioceptive trend for judgments to be made in the direction of the transformed visual input, i.e., to become less accurate in objective terms.

Discussion. The data from the experiment show that there is no difference in reports of the spatial position of an object for the two experimental conditions, each at two levels of transformed visual input.

The conditions where $S$ has a greater variety of proprioceptive activity prior to making a spatial judgment do not lead to greater accuracy in objective terms as Over (1966) suggested. This is consistent with the concept of visual capture and demonstrates that the "spatial" position of the object as it is signalled through information from the muscle and joint receptors is dominated by the transformed visual information.

The data also show that, although contact with the object is achieved each time, the reports of the spatial position of the object become less accurate in objective terms, i.e., visual dominance does not diminish over trials. On the

Table 1

Mean Differences Between Pretests and Each of Ten Exposure Trials for All Four Experimental Conditions

Trials

\begin{tabular}{crcccccccccc} 
Conditions & \multicolumn{1}{c}{1} & 2 & 3 & 4 & 5 & 6 & 7 & 8 & 9 & 10 & Means \\
\hline A 20 & 13.7 & 12.5 & 13.8 & 13.0 & 15.8 & 13.3 & 15.2 & 14.5 & 17.0 & 15.0 & 14.36 \\
B 20 & 9.9 & 13.6 & 12.0 & 13.5 & 11.6 & 11.1 & 12.6 & 13.6 & 11.4 & 13.8 & 12.28 \\
A 40 & 18.6 & 20.1 & 20.4 & 20.4 & 22.3 & 23.6 & 23.5 & 25.2 & 24.7 & 23.6 & 22.23 \\
B 40 & 13.4 & 12.7 & 13.8 & 16.1 & 15.6 & 18.4 & 16.1 & 18.2 & 18.2 & 17.0 & 15.98 \\
\hline
\end{tabular}


contrary, in three of the four conditions there was a significant increase in the degree to which later responses corresponded with the transformed visual input. With reference to the objective spatial position this can be regarded as an increasing intermodal illusion. This is consistent with Thurner (1967), who reports an increasing suppression of kinesthetic position signals induced by active performance under visual displacement.

The increase in the intermodal illusion in the present experiment can be explained in terms of the aftereffect or persistence of response. Previous work (Held \& Freedman, 1963; Singer \& Day, 1966) has shown that the responses of behavioral compensation persist after the removal of the optical device provided there is no other visual feedback. It is possible that this response persistence from the earlier trials is additive and increases the magnitude of the response on later trials, resulting in the observed increase in the illusion; i.e., persistence of the behavioral compensation response which was produced by the first trial is still operative during subsequent trials and thus adds to the magnitude of the behavioral compensation responses at those trials. This additive effect will sum over trials.

It would be interesting to extend this work to different degrees of transformation, since Over's (1966) data, as well as Thurner's (1967) suggest that different factors seem to operate as the discordance between the visual and proprioceptive input increases.

\section{REFERENCES}

COLLINS, J. K., \& SINGER, G. Behavioral compensation in the measurement of sensory spatial aftereffects, $J$. exp. Psychol, in press.

DAY, R. H., \& SINGER, G. Sensory adaptation and behavioral compensation with spatially transformed vision and hearing, Psychol. Bull, 1967, 67, 307-322.

HARRIS, C. S. Perceptual adaptation to inverted, reversed, and displaced vision, Psychol Rev., 1965, 72, 419-444.

HELD, R., \& FREEDMAN, S. J. Plasticity in human sensorimotor control, Science, 1963, 142, 455-462.

OVER, R. An experimentally induced conflict between vision and proprioception, Brit. J. PsychoL, 1966, 57, 335-341.

SINGER, G., \& DAY, R. H. The effects of spatial judgments on the perceptual aftereffect resulting from transformed vision, Aust. J. PsychoL, $1966,18,63-70$.

THURNER, F. Studies of artificially disturbed sensory coordination in man. Innsbruck: Institut für Psychologie der Universităt Innsbruck, 1967. 